Research Article

\title{
Metric Dimension on Path-Related Graphs
}

\author{
Saqib Nazeer (D), ${ }^{1}$ Muhammad Hussain (D, ${ }^{1}$ Fatimah Abdulrahman Alrawajeh, ${ }^{2}$ \\ and Sultan Almotairi $\mathbb{D i}^{3}$ \\ ${ }^{1}$ Department of Mathematics, COMSATS University Islamabad, Lahore Campus, Lahore 54000, Pakistan \\ ${ }^{2}$ Department of Mathematics, Faculty of Science College, Imam Abdulrahman Bin Faisal University (IAU), \\ Dammam 31441, Saudi Arabia \\ ${ }^{3}$ Department of Natural and Applied Sciences, Faculty of Community College, Majmaah University, \\ Majmaah 11952, Saudi Arabia
}

Correspondence should be addressed to Sultan Almotairi; almotairi@mu.edu.sa

Received 30 September 2021; Accepted 15 October 2021; Published 29 October 2021

Academic Editor: Tabasam Rashid

Copyright (C) 2021 Saqib Nazeer et al. This is an open access article distributed under the Creative Commons Attribution License, which permits unrestricted use, distribution, and reproduction in any medium, provided the original work is properly cited.

Graph theory has a large number of applications in the fields of computer networking, robotics, Loran or sonar models, medical networks, electrical networking, facility location problems, navigation problems etc. It also plays an important role in studying the properties of chemical structures. In the field of telecommunication networks such as CCTV cameras, fiber optics, and cable networking, the metric dimension has a vital role. Metric dimension can help us in minimizing cost, labour, and time in the above discussed networks and in making them more efficient. Resolvability also has applications in tricky games, processing of maps or images, pattern recognitions, and robot navigation. We defined some new graphs and named them $s$-middle graphs, $s$-total graphs, symmetrical planar pyramid graph, reflection symmetrical planar pyramid graph, middle tower path graph, and reflection middle tower path graph. In the recent study, metric dimension of these path-related graphs is computed.

\section{Introduction and Preliminary Results}

Term metric dimension was firstly revealed by mathematician named Slater [1]. He explained the study of sonar or Loran model station by this concept. Harary, another mathematician, with the help of Melter, independently described the same concept of metric dimension [2].

Consider $G$ is a simple, undirected, and finite graph and $T=\left\{s_{1}, s_{2}, \ldots, s_{f}\right\}$ is ordered subset of vertex set of $G$, so $r(s \mid T)$ is a representation of the vertex $s$ with respect to $T$ which is $f$-tuple $\left(d\left(l, s_{1}\right), d\left(l, s_{2}\right), d\left(l, s_{3}\right), \ldots, d\left(l, s_{f}\right)\right\}$. If each element of vertex set possesses unique representation with $T$, then we conclude that $T$ is resolving set of $S$. If $T$ is the smallest resolving set for $G$, then its cardinality becomes metric dimension of $G$ and can be symbolized as $\operatorname{dim}(G)$.

Path graph is the only graph with metric dimension 1 [3]. Metric dimension of cycle graph is 2. Resolvability also has applications in tricky games, processing of maps or images, pattern recognitions, and networking robot navigation [4]. More details of applications of metric dimension can be seen in [5-10]. It is suggested to study the following references for better comprehension and details of metric dimension of graphs [11-16].

A lot of work has been carried out by many authors on metric dimension of path-related graphs. In 2012, Ali et al. computed constant metric dimension of middle path graph and power of path graph [10]. In 2017, Alholi et al. also put contribution in this field and found constant metric dimension [17]. Shahida and Sunitha found constant metric dimension of join of any two graphs [18]. In 2016, Peng et al. calculated metric dimension of Kenser graphs [19].

Ali et al., in 2012, worked on metric dimension of total graph of path graph and found it to be constant [10]. In 2019, Pan et al. calculated the unbounded metric dimension of splitting graphs of path graph and cycle graph [20]. In 2021, Nawaz et al. proved that metric dimension of path power three and four is unbounded; also, they proved some results on edges of power of path and power of total graph [21].

Our research is in continuation with their work, and we computed the metric dimension of $s$-middle path graphs and 
$s$-total path graphs. These graphs have bounded metric dimension. In the paper under study, we also calculated metric dimension of other path-related graphs such as middle tower path graph, reflection middle tower path graph, symmetrical planar pyramid path graph, and reflection symmetrical planar pyramid path graph.

1.1. Metric Dimension of s-Middle Graph. We generalized the concept of middle of a graph and extended this idea to $s$ vertices. We took two particular cases for path graph and with the help of these results and finally computed the metric dimension of $s$-middle graph of the path graph.

Definition 1. For undirected simple (planar or nonplanar) graph $G$ with $V(G)$ and $E(G)$ as vertex and edge set, respectively, $\quad V(G)=\left\{v_{1}, v_{2}, \ldots, v_{l}\right\} \quad$ and $E(G)=\left\{e_{1}, e_{2}, \ldots, e_{u}\right\}$. We associate for every edge in edge set $s$ vertices, forming another graph possessing vertex set represented by $V(s-\operatorname{Mid}(G))$ which is given by $V(s-\operatorname{Mid}(G))=V(G) \bigcup\left(\cup_{u=1}^{s} V^{u}\right)$, and if there are $l$ vertices in $G$ and number of edges is $u$, then $s-\operatorname{Mid}(G)$ contains $l+s u$ vertices. Each $v_{g}$ in $V(s-\operatorname{Mid}(G))$ is adjacent to $v_{h}$ in $V(s-\operatorname{Mid}(G))$ :

(1) $v_{g} \sim v_{g}^{u}$ if $v_{g}$ is incident to $e_{g}$ in $G$

(2) $v_{g}^{u} \sim v_{f}^{u}$ if $e_{g}$ is incident to $e_{f}$ in $G$

Example 1. For a particular case of $s$-Mid $(G)$, we dicussed two-middle path graph in which exactly two vertices were assigned against each edge. We take $G=P_{q}$ (path graph containing $q$ vertices). We associate $h_{l}$ and $s_{l}$ so that $V^{*}=\left\{h_{1}, h_{2}, h_{3}, \ldots, h_{q-1}\right\}, V^{* *}=\left\{s_{1}, s_{2}, s_{3}, \ldots, s_{q-1}\right\}$.

Thus, the vertex set of $\operatorname{Two}-\operatorname{Mid}\left(P_{q}\right)$ is $V\left(\right.$ Two $\left.-\operatorname{Mid}\left(P_{q}\right)\right)=V\left(P_{q}\right) \cup V^{*}\left(P_{q}\right) \cup V^{* *}\left(P_{q}\right)$. Each $v_{l} \sim v_{m}$ in Two $-\operatorname{Mid}\left(P_{q}\right)$ :

(1) $v_{l} \sim s_{i}$ and $v_{l} \sim h_{l}$ are incident to $e_{l}$ in $P_{q}$

(2) $s_{l} \sim s_{m}$ and $h_{l} \sim h_{m}$ if and only if $e_{i}$ is adjacent to $e_{m}$ in $P_{q}$

Two-middle path graph and three-middle path graph are explained in Figures 1 and 2.

Theorem 1. $\operatorname{dim}\left(\operatorname{Two}-\operatorname{Mid}\left(p_{q}\right)\right)$ is 2, where $q \geq 3$.

Proof. Two-Mid $\left(P_{q}\right)$ is not path when $q \geq 3$, so its metric dimension must be greater than 1 . The vertex set for Two-Mid $\left(P_{q}\right)$ is $V\left(\right.$ Two $\left.-\operatorname{Mid}\left(P_{q}\right)\right)$ and is given by $V\left(\right.$ Two $\left.-\operatorname{Mid}\left(P_{q}\right)\right)=\left\{v_{1}, v_{2}, v_{3}, \ldots, v_{q}\right\}$ $\bigcup\left\{h_{1}, h_{2}, h_{3}, \ldots, h_{q-1}\right\} \bigcup\left\{s_{1}, s_{2}, s_{3}, \ldots, s_{q-1}\right\}$.

Assume $W=\left\{s_{1}, s_{q-1}\right\}$ as a resolving set for Two-Mid $\left(P_{q}\right)$ so that

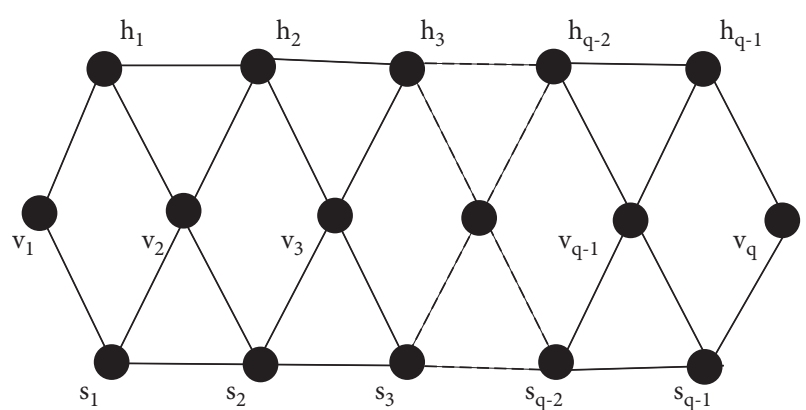

FIgURE 1: Two-middle path graph.

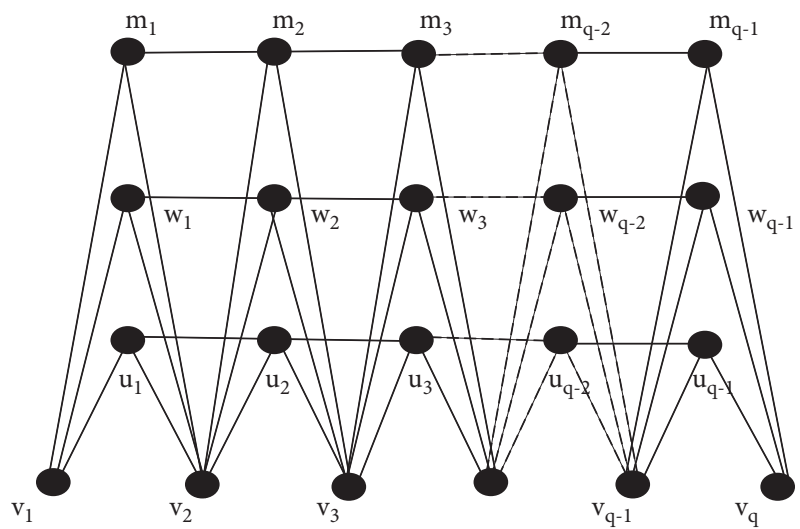

Figure 2: Three-middle path graph.

$$
\begin{aligned}
& r\left(v_{l} \mid W\right)= \begin{cases}(l, q-1), & l=1, \\
(l-1, q-l), & l=2,3,4, \ldots, q-1, \\
(l-1,1), & l=q,\end{cases} \\
& r\left(s_{j} \mid W\right)=(j-1, q-j-1), \quad \forall j=1,2,3, \ldots, q-1,
\end{aligned}
$$

When $l \neq g$ and $v_{l}$ and $v_{g}$ have the same representation for $W$, contradiction arises, i.e.,

$$
\begin{aligned}
r\left(v_{l} \mid W\right) & =r\left(v_{g} \mid W\right) \\
\Rightarrow(l-1, q-l) & =(g-1, q-g) \\
\Rightarrow l & =g(\text { contradiction }) .
\end{aligned}
$$

Similarly, when $l \neq g$,

$$
\begin{aligned}
r\left(s_{l} \mid W\right) & =r\left(s_{g} \mid W\right) \\
\Rightarrow(l-1, q-l-1) & =(g-1, q-g-1) \\
\Rightarrow 1 & =g(\text { contradiction }) .
\end{aligned}
$$


When $l \neq g$,

$$
\begin{aligned}
r\left(h_{l} \mid W\right) & =r\left(h_{q} \mid W\right) \\
\Rightarrow(l, q-l) & =(g-1, q-g-1) \\
\Rightarrow 1=g-1 \text { and } 1 & =g+1(\text { contradiction }) .
\end{aligned}
$$

When $l \neq g$,

$$
\begin{aligned}
r\left(v_{l} \mid S\right) & =r\left(s_{g} \mid S\right) \\
\Rightarrow(l, q-l) & =(g, g-l) \\
\Rightarrow 1 & =g(\text { contradiction }) .
\end{aligned}
$$

When $l \neq g$,

$$
\begin{aligned}
r\left(h_{l} \mid W\right) & =r\left(s_{g} \mid W\right) \\
\Rightarrow(l, q-l) & =(g, q-1) \\
\Rightarrow 1 & =g \text { (contradiction). }
\end{aligned}
$$

Hence, Two-Mid $\left(P_{q}\right)$ possesses $W$ as its smallest resolving set, so its metric dimension is 2 .

Theorem 2. $\operatorname{dim}\left(\right.$ Three $\left.-\operatorname{Mid}\left(P_{q}\right)\right)$ is 3, where $q \geq 3$.

Proof. The vertex set for Three $-\operatorname{Mid}\left(P_{q}\right)$ is $V$ (Three $\left.\operatorname{Mid}\left(P_{q}\right)\right)$ and is given by

$$
\begin{aligned}
V\left(\text { Three }-\operatorname{Mid}\left(P_{q}\right)\right) & =\left\{v_{1}, v_{2}, v_{3}, \ldots, v_{q}\right\} \\
& \bigcup\left\{u_{1}, u_{2}, u_{3}, \ldots, u_{q-1}\right\} \\
& \bigcup\left\{w_{1}, w_{2}, w_{3}, \ldots, w_{q-1}\right\} \\
& \bigcup\left\{m_{1}, m_{2}, m_{3}, \ldots, m_{q-1}\right\} .
\end{aligned}
$$

As Three $-\operatorname{Mid}\left(P_{q}\right)$ is not a path graph, so it possesses metric dimension greater than 1 .

Assume $W=\left\{u_{1}, v_{1}, m_{1}\right\}$ as a resolving set for Three $\operatorname{Mid}\left(P_{q}\right)$ so that

$$
\begin{aligned}
& r\left(v_{g} \mid W\right)= \begin{cases}(1,0,1), & g=1, \\
(g-1, g, g-1), & g=2,3,4, \ldots, q,\end{cases} \\
& r\left(u_{g} \mid W\right)= \begin{cases}(0,1,2), & g=1, \\
(g-1, g, g), & g=2,3,4, \ldots, q-1,\end{cases} \\
& r\left(w_{g} \mid W\right)= \begin{cases}(2,1,2), & g=1, \\
(g, g, g), & g=2,3,4, \ldots, q-1,\end{cases} \\
& r\left(m_{g} \mid W\right)= \begin{cases}(2,1,0), & g=1, \\
(g, g, g-1), & g=2,3,4, \ldots, q-1 .\end{cases}
\end{aligned}
$$

When $l \neq g$ and $v_{l}$ and $v_{g}$ have the same representation with respect to $w$, a contradiction arises, i.e.,

$$
\begin{aligned}
r\left(v_{l} \mid W\right) & =r\left(v_{g} \mid W\right) \\
\Rightarrow(l-1, l, l-1) & =(g-1, q, g-1) \\
\Rightarrow l & =g(\text { contradiction }) .
\end{aligned}
$$

Similarly, when $l \neq g$,

$$
\begin{aligned}
r\left(u_{l} \mid W\right) & =r\left(u_{g} \mid W\right) \\
\Rightarrow(l-1, l, l) & =(g-1, g, g) \\
\Rightarrow l & =g \text { (contradiction). }
\end{aligned}
$$

When $l \neq g$,

$$
\begin{aligned}
r\left(w_{l} \mid W\right) & =r\left(w_{g} \mid W\right) \\
\Rightarrow(l, l, l) & =(g, g, g) \\
\Rightarrow l & =g \text { (contradiction). }
\end{aligned}
$$

When $l \neq g$,

$$
\begin{aligned}
r\left(m_{l} \mid W\right) & =r\left(m_{g} \mid W\right) \\
\Rightarrow(l, l, l-l) & =(g, g, g-1) \\
\Rightarrow l & =g(\text { contradiction }) .
\end{aligned}
$$

When $l \neq g$,

$$
\begin{aligned}
r\left(v_{l} \mid W\right) & =r\left(u_{g} \mid W\right) \\
\Rightarrow(l-1, l, l-1) & =(g-1, g, g) \\
\Rightarrow l & =g \text { (contradiction). }
\end{aligned}
$$

When $l \neq g$,

$$
\begin{aligned}
r\left(v_{l} \mid W\right) & =r\left(w_{g} \mid W\right) \\
\Rightarrow(l-1, l, l-l) & =(g, g, g) \\
\Rightarrow l & =g(\text { contradiction }) .
\end{aligned}
$$

When $l \neq g$,

$$
\begin{aligned}
r\left(u_{l} \mid W\right) & =r\left(w_{g} \mid W\right) \\
\Rightarrow(l-1, l, l-1) & =(g, g, g) \\
\Rightarrow l & =g(\text { contradiction }) .
\end{aligned}
$$

When $l \neq g$,

$$
\begin{aligned}
r\left(u_{l} \mid W\right) & =r\left(w_{g} \mid W\right) \\
\Rightarrow(l-1, l, l) & =(g, g, g) \\
\Rightarrow l & =g \text { (contradiction). }
\end{aligned}
$$

When $l \neq g$,

$$
\begin{aligned}
r\left(u_{l} \mid W\right) & =r\left(m_{g} \mid W\right) \\
\Rightarrow(l-1, l, l) & =(g, g, g-1) \\
\Rightarrow l & =g(\text { contradiction }) .
\end{aligned}
$$

When $l \neq g$,

$$
\begin{aligned}
r\left(w_{l} \mid W\right) & =r\left(m_{g} \mid W\right) \\
\Rightarrow(l, l, l) & =(g, g, g-1) \\
\Rightarrow l & =g(\text { contradiction }) .
\end{aligned}
$$

Hence, $W$ is the resolving set for Three $-\operatorname{Mid}\left(P_{q}\right)$. Now, we shall show that no resolving set having cardinality 2 exists. 
Let $T_{1}=\left\{v_{l}, v_{g}\right\}$ be metric basis of Three $-\operatorname{Mid}\left(P_{q}\right)$; then, $r\left(u_{j} \mid T_{1}\right)=r\left(m_{j} \mid T_{1}\right)=r\left(w_{j} \mid T_{1}\right)$, so uniqueness is violated.

Let $T_{2}=\left\{u_{l}, u_{g}\right\}$ be metric bases of Three $-\operatorname{Mid}\left(P_{q}\right)$; then, $r\left(w_{j} \mid T_{2}\right)=r\left(m_{j} \mid T_{2}\right)$, so uniqueness is violated.

Let $T_{3}=\left\{w_{l}, w_{g}\right\}$ be metric bases of Three $-\operatorname{Mid}\left(P_{q}\right)$; then, $r\left(u_{j} \mid T_{3}\right)=r\left(m_{j} \mid T_{3}\right)$, so uniqueness is violated. Let $T_{4}=\left\{m_{l}, m_{g}\right\}$ be metric bases of Three $-\operatorname{Mid}\left(P_{q}\right)$; then, $r\left(w_{j} \mid T_{4}\right)=r\left(u_{j} \mid T_{4}\right)$, so uniqueness is violated. Let $T_{5}=\left\{v_{l}, w_{g}\right\}$ be metric bases of Three $-\operatorname{Mid}\left(P_{q}\right)$; then, $r\left(u_{j} \mid T_{5}\right)=r\left(m_{j} \mid T_{5}\right)$, so uniqueness is violated. Let $T_{6}=\left\{v_{l}, u_{g}\right\}$ be metric bases of Three $-\operatorname{Mid}\left(P_{q}\right)$; then, $r\left(w_{j} \mid T_{6}\right)=r\left(m_{j} \mid T_{6}\right)$, so uniqueness is violated. Let $T_{7}=\left\{v_{l}, m_{g}\right\}$ be metric bases of Three $-\operatorname{Mid}\left(P_{q}\right)$; then, $r\left(w_{j} \mid T_{7}\right)=r\left(u_{j} \mid T_{7}\right)$, so uniqueness is violated. Let $T_{8}=\left\{u_{l}, w_{g}\right\}$ be metric bases of Three $-\operatorname{Mid}\left(P_{q}\right)$; then, $r\left(v_{j} \mid T_{8}\right)=r\left(m_{j-1} \mid T_{8}\right)$, so uniqueness is violated.
Let $T_{9}=\left\{u_{l}, m_{g}\right\}$ be metric bases of Three $-\operatorname{Mid}\left(P_{q}\right)$; then, $r\left(v_{j} \mid T_{9}\right)=r\left(m_{j-1} \mid T_{9}\right)$, so uniqueness is violated. Let $T_{10}=\left\{w_{l}, m_{g}\right\}$ be metric bases of Three $-\operatorname{Mid}\left(P_{q}\right)$; then, $r\left(v_{j} \mid T_{10}\right)=r\left(u_{j-1} \mid T_{10}\right)$, so uniqueness is violated.

So, there are no metric bases of order 2 . Hence, $W$ is the resolving set of order 3 and metric dimension of Three $\operatorname{Mid}\left(P_{q}\right)$ is 3 .

Theorem 3. Metric dimension of s-Middle $\left(P_{q}\right)$ is $s$.

Proof. The vertex set of $s$-Middle $\left(P_{q}\right)$ is $\left\{v_{1}, v_{2}, v_{3}, \ldots, v_{p}\right\} \bigcup\left\{w_{g}^{m}\right\}$, where $g=1,2,3, \ldots, p-1$ and $m=1,2,3, \ldots, s$. Let $W=\left\{w_{1}^{1}, w_{1}^{2}, w_{1}^{3}, \ldots, w_{1}^{s}\right\}$ be the resolving set of the $s$-Middle $\left(P_{q}\right)$; then, $r\left(v_{g} \mid W\right)=(g, g-1, g-1, \ldots, g-1)_{(s-\text { tuple })}, \quad$ where $g=1,2,3, \ldots, p$ :

$$
r\left(w^{(h)_{g}} \mid W\right)= \begin{cases}(g-1, g, g, \ldots, g)_{(s-\text { tuple })} & g=1,2,3, \ldots, p-1 ; h=1, \\ (g, g-1, g, \ldots, g)_{(s-\text { tuple })} & g=1,2,3, \ldots, p-1 ; h=2, \\ (g, g, g-1, \ldots, g)_{(s-\text { tuple })}, & g=1,2,3, \ldots, p-1 ; h=3, \\ \cdot & \\ (g, g, g, \ldots, g-1)_{(s-\text { tuple })}, & g=1,2,3, \ldots, p-1 ; h=s,\end{cases}
$$

When $l \neq m$ and $v_{l}$ and $v_{m}$ possess the same representation for $W$, a contradiction arises, i.e.,

$$
\begin{aligned}
r\left(v_{l} \mid W\right) & =r\left(v_{m} \mid W\right) \\
& \Rightarrow(l, l-1, l-1, \ldots, l-1)_{(s-\text { tuple })} \\
& =(m, m-1, m-1, \ldots, m-1)_{(s-\text { tuple })} \\
& \Rightarrow l=m(\text { contradiction }) .
\end{aligned}
$$

Similarly, when $l \neq m$,

$$
\begin{aligned}
r\left(w_{l}^{h} \mid W\right) & =r\left(w_{m}^{h} \mid W\right) \\
& \Rightarrow\left(l, l, l, \ldots, l-1_{h^{\text {th }}}\right. \text { position } \\
& =(m, \ldots, l)_{(s-\text { tuple })} \\
& \Rightarrow l=m, \ldots, m-1_{h^{\text {th }}} \text { position } \\
& =m, \ldots, m)_{(s \text {-tuple })} \\
&
\end{aligned}
$$

$$
\begin{aligned}
r\left(w_{g}^{l} \mid W\right) & =r\left(w_{g}^{m} \mid W\right) \\
& \Rightarrow\left(g, g, g, \ldots, g-1_{l^{\text {th }} \text { position }}, g, \ldots, g\right)_{(s-\text { tuple })} \\
& =\left(g, g, g, \ldots, g-1_{m^{\text {th }} \text { position }}, g, \ldots, g\right)_{(s-\text { tuple })} \\
& \Rightarrow g-1=g(\text { contradiction }) .
\end{aligned}
$$

When $l \neq m$,

$$
\begin{aligned}
u \neq v r\left(w_{l}^{u} \mid W\right) & =r\left(w_{m}^{v} \mid W\right) \\
& \Rightarrow\left(l, l, l, \ldots, l-1_{\left(u^{\text {th }} \text { position }\right)}, l \ldots, l\right)_{(s-\text { tuple })} \\
& =\left(m, m, m, \ldots, m-1_{\left(v^{\text {th }} \text { position }\right)}, m, \ldots, m\right)_{(s-\text { tuple })} \\
& \Rightarrow l-1=m \text { and } l=m-1 \text { (contradiction) } .
\end{aligned}
$$

When $l \neq m$, 
Now, we shall show that there is no resolving set with $s-1$ elements that exists. So, on the contrary, let a set with $s-1$ elements be a resolving set for $s$-Middle $P_{q}$. Then, particularly, for $s=3$, there exists a resolving set of $3-1=2$ elements. So, metric dimension of Three-Middle $P_{q}$ is not 2, and it leads to contradiction.

So, there is no resolving set with $s-1$ elements, and hence, $s$-Middle $\left(P_{q}\right)$ has metric dimension $s$.

1.2. s-Total Graph of a Path. We discuss here more generalized concept of total graph of some graphs. We shall apply the very concept for $s=2,3$ on the path graph and use these results to find metric dimension of $s$-total graph of the path graph.

Definition 2. For undirected simple (planar or nonplanar) graph $G$ with vertex set $V(G)$ and $E(G)$ as the edge set, $V(G)=\left\{v_{1}, v_{2}, \ldots, v_{s}\right\}$ and $E(G)=\left\{e_{1}, e_{2}, \ldots, e_{u}\right\}$. We associate for every edge in edge set $s$ vertices, forming another graph possessing vertex set represented by $V(s-T(G))$ which is given by $V(s-T(G))=V(G) \cup\left(\cup_{u=1}^{s} V^{u}\right)$, and if the total number of vertices in $G$ is $r$ and number of edges is $u$, then $s$-Total $(G)$ contains $r+s u$ vertices. Each $v_{g}$ in $V(s-$ $T(G))$ is adjacent to $v_{h}$ in $V(s-T(G))$ :

(1) $v_{g} \sim v_{f}$ if $v_{g} \sim v_{f}$ in $G$

(2) $v_{g} \sim v_{g}^{u}$ if $v_{g}$ is incident to $e_{g}$ in $G$

(3) $v_{g}^{u} \sim v_{f}^{u}$ if $e_{g}$ is incident to $e_{f}$ in $G$

Two-total graph of a path and Three-total graph of a path are explained in Figures 3 and 4.

Theorem 4. $\operatorname{dim}\left(T w o-T\left(p_{q}\right)\right.$ is 2 , where $q \geq 2$.

Proof. Two $-T\left(P_{q}\right)$ is not the path when $q \geq 2$, so its metric dimension must be greater than 1 . The vertex set for Two $T\left(P_{q}\right)$ is $V$ (Two $-T\left(P_{q}\right)$ ) and is given by $V$ (Two $-T\left(P_{q}\right)$ ) $=\left\{v_{1}, v_{2}, v_{3}, \ldots, v_{q}\right\} \bigcup\left\{h_{1}, h_{2}, h_{3}, \ldots, h_{q-1} \bigcup\left\{s_{1}, s_{2}, s_{3}, \ldots\right.\right.$, $\left.s_{q-1}\right\}$.

Assume $W=\left\{h_{1}, h_{q-1}\right\}$ as a resolving set for Two $-T\left(P_{q}\right)$ so that

$$
\begin{aligned}
& r\left(v_{l} \mid W\right)= \begin{cases}(l, q-1), & j=1, \\
(l-1, q-l), & j=2,3,4, \ldots, q-1, \\
(l-1,1), & l=q,\end{cases} \\
& r\left(s_{h} \mid W\right)=(l-1, q-l-1) \quad \forall j=1, \ldots, q-1 \text {, } \\
& r\left(h_{j} \mid W\right)= \begin{cases}(2, q-1) & l=1, \\
(l, q-l), & q=2,3,4, \ldots, q-2, \\
(l, 2), & l=q-1 .\end{cases}
\end{aligned}
$$

When $u \neq r$ and $h_{u}$ and $h_{r}$ possess the same representation for $W$, a contradiction arises, i.e.,

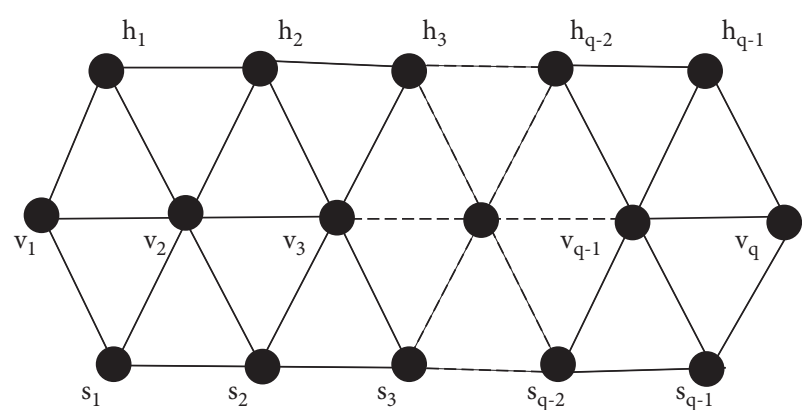

FIgURE 3: Two-total graph of the path graph.

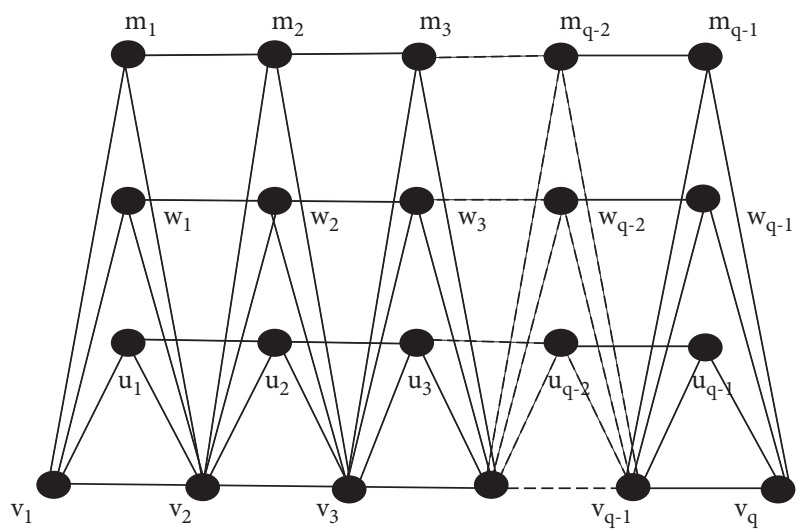

FIGURE 4: Three-total graph of the path graph.

$$
\begin{aligned}
r\left(h_{u} \mid W\right) & =r\left(h_{r} \mid W\right) \\
\Rightarrow(u-1, q-u-1) & =(r-1, q-r) \\
\Rightarrow l & =g(\text { contradiction }) .
\end{aligned}
$$

Similarly, when $u \neq r$,

$$
\begin{aligned}
r\left(s_{u} \mid W\right) & =r\left(s_{r} \mid W\right) \\
\Rightarrow(u-1, q-u) & =(r-1, q-r) \\
\Rightarrow u & =r(\text { contradiction }) .
\end{aligned}
$$

When $u \neq r$,

$$
\begin{aligned}
r\left(h_{u} \mid W\right) & =r\left(s_{r} \mid W\right) \\
\Rightarrow(u, q-u) & =(r, q-r) \\
\Rightarrow u & =r \text { (contradiction). }
\end{aligned}
$$

When $u \neq r$,

$$
\begin{aligned}
r\left(v_{u} \mid W\right) & =r\left(v_{r} \mid W\right) \\
\Rightarrow(u-l, q-u) & =(r-1, q-r) \\
\Rightarrow u & =r(\text { contradiction }) .
\end{aligned}
$$

Hence, Two $-T\left(P_{q}\right.$ possesses $W$ as its smallest resolving set, so its metric dimension is 2 .

Theorem 5. $\operatorname{dim}\left(\right.$ Three $-T\left(p_{q}\right)$ is 3 , where $q \geq 3$. 
Proof. The vertex set for Three $-T\left(P_{q}\right)$ is $V\left(\right.$ Three $\left.-T\left(P_{q}\right)\right)$ and is given by $V\left(\right.$ Three $\left.-T\left(P_{q}\right)\right)$ $=\left\{v_{1}, v_{2}, v_{3}, \ldots, v_{q}\right\} \bigcup\left\{u_{1}, u_{2}, u_{3}, \ldots, u_{q-1} \bigcup\left\{w_{1}, w_{2}, w_{3}\right.\right.$, $\left.\ldots, w_{q-1}\right\} \bigcup\left\{m_{1}, m_{2}, m_{3}, \ldots, m_{q-1}\right\}$. As Three $-T\left(P_{q}\right)$ is not a path graph, so it possesses metric dimension greater than 1 .

Assume $W=\left\{u_{1}, v_{1}, m_{1}\right\}$ as a resolving set for Three $T\left(P_{q}\right)$ so that

$$
\begin{aligned}
& r\left(v_{g} \mid W\right)= \begin{cases}(1,0,1), & g=1, \\
(g-1, g-1, g-1), & g=2,3,4, \ldots, q-1,\end{cases} \\
& r\left(u_{g} \mid W\right)= \begin{cases}(0,1,2), & g=1, \\
(g-1, g, g), & g=2,3,4, \ldots, q-1,\end{cases} \\
& r\left(w_{g} \mid W\right)= \begin{cases}(2,1,2), & g=1, \\
(g, g, g), & g=2,3,4, \ldots, q-1,\end{cases} \\
& r\left(m_{g} \mid W\right)= \begin{cases}(2,1,0), & g=1, \\
(g, g, g-1), & g=2,3,4, \ldots, q-1 .\end{cases}
\end{aligned}
$$

When $l \neq g$ and $v_{l}$ and $v_{g}$ possess the same representation for $W$, a contradiction arises, i.e.,

$$
\begin{aligned}
r\left(v_{l} \mid W\right) & =r\left(v_{g} \mid W\right) \\
\Rightarrow(l-1, l, l-1) & =(g-1, g-1, g-1) \\
\Rightarrow l & =g \text { (contradiction). }
\end{aligned}
$$

Similarly, when $g \neq l$,

$$
\begin{aligned}
r\left(u_{g} \mid W\right) & =r\left(u_{l} \mid W\right) \\
\Rightarrow(g-1, g, g) & =(l-1, l, l) \\
\Rightarrow g & =l \text { (contradiction). }
\end{aligned}
$$

When $l \neq g$,

$$
\begin{aligned}
r\left(w_{l} \mid W\right) & =r\left(w_{g} \mid W\right) \\
\Rightarrow(l, l, l) & =(g, g, g) \\
\Rightarrow l & =g \text { (contradiction). }
\end{aligned}
$$

When $l \neq g$,

$$
\begin{aligned}
r\left(m_{l} \mid W\right) & =r\left(m_{g} \mid W\right) \\
\Rightarrow(l, l, l-l) & =(g, g, g-1) \\
\Rightarrow l & =g(\text { contradiction }) .
\end{aligned}
$$

When $l \neq g$,

$$
\begin{aligned}
r\left(v_{l} \mid W\right) & =r\left(u_{g} \mid W\right) \\
\Rightarrow(l-1, l-1, l-1) & =(g-1, g, g) \\
\Rightarrow l & =g(\text { contradiction }) .
\end{aligned}
$$

When $l \neq g$,

$$
\begin{aligned}
r\left(u_{l} \mid W\right) & =r\left(w_{g} \mid W\right) \\
\Rightarrow(l-1, l, l) & =(g, g, g) \\
\Rightarrow l & =g \text { (contradiction). }
\end{aligned}
$$

When $l \neq g$,

$$
\begin{aligned}
r\left(u_{l} \mid W\right) & =r\left(m_{g} \mid W\right) \\
\Rightarrow(l-1, l, l) & =(g, g, g-1) \\
\Rightarrow l & =g(\text { contradiction }) .
\end{aligned}
$$

When $l \neq g$,

$$
\begin{aligned}
r\left(w_{l} \mid W\right) & =r\left(m_{g} \mid W\right) \\
\Rightarrow(l, l, l) & =(g, g, g-1) \\
\Rightarrow l & =g \text { (contradiction). }
\end{aligned}
$$

Hence, $W$ is resolving set for Three-Total $\left(P_{q}\right)$. Now, we shall show that no resolving set having cardinality 2 exists.

Let $T_{1}=\left\{v_{l}, v_{g}\right\}$ be a resolving set of Three $-T\left(P_{q}\right)$; then, $r\left(u_{j} \mid T_{1}\right)=r\left(m_{j} \mid T_{1}\right)=r\left(w_{j} \mid T_{1}\right)$, so uniqueness is violated.

Let $T_{2}=\left\{u_{l}, u_{g}\right\}$ be metric bases of Three $-T\left(P_{q}\right)$; then, $r\left(w_{j} \mid T_{2}\right)=r\left(m_{j} \mid T_{2}\right)$, so uniqueness is violated. Let $T_{3}=\left\{w_{l}, w_{g}\right\}$ be metric bases of Three $-T\left(P_{q}\right)$; then, $r\left(u_{j} \mid T_{3}\right)=r\left(m_{j} \mid T_{3}\right)$, so uniqueness is violated.

Let $T_{4}=\left\{m_{l}, m_{g}\right\}$ be metric bases of Three $-T\left(P_{q}\right)$; then, $r\left(w_{j} \mid T_{4}\right)=r\left(u_{j} \mid T_{4}\right)$, so uniqueness is violated.

Let $T_{5}=\left\{v_{l}, w_{g}\right\}$ be metric bases of Three $-T\left(P_{q}\right)$; then, $r\left(u_{j} \mid T_{5}\right) \stackrel{=}{=}\left(m_{j} \mid T_{5}\right)$, so uniqueness is violated.

Let $T_{6}=\left\{v_{l}, u_{g}\right\}$ be metric bases of Three $-T\left(P_{q}\right)$; then, $r\left(w_{j} \mid T_{6}\right)=r\left(m_{j} \mid T_{6}\right)$, so uniqueness is violated.

Let $T_{7}=\left\{v_{l}, m_{g}\right\}$ be metric bases of Three $-T\left(P_{q}\right)$; then, $r\left(w_{j} \mid T_{7}\right)=r\left(u_{j} \mid T_{7}\right)$, so uniqueness is violated.

Let $T_{8}=\left\{u_{l}, w_{g}\right\}$ be metric bases of Three $-T\left(P_{q}\right)$; then, $r\left(v_{j} \mid T_{8}\right)=r\left(m_{j-1} \mid T_{8}\right)$, so uniqueness is violated. Let $T_{9}=\left\{u_{l}, m_{g}\right\}$ be metric bases of Three $-T\left(P_{q}\right)$; then, $r\left(v_{j} \mid T_{9}\right)=r\left(w_{j-1} \mid T_{9}\right)$, so uniqueness is violated. Let $T_{10}=\left\{w_{l}, m_{g}\right\}$ be metric bases of Three $-T\left(P_{q}\right)$; then, $r\left(v_{j} \mid T_{10}\right)=r\left(u_{j-1} \mid T_{10}\right)$, so uniqueness is violated.

So, there are no metric bases of order 2 . Hence, $W$ is the resolving set of order 3 and metric dimension of Three $T\left(P_{q}\right)$ is 3 .

Theorem 6. Metric dimension of $s-$ Total $\left(P_{q}\right)$ is $s$.

Proof. The vertex set of $s$-Total $\left(P_{q}\right)$ is $\left\{v_{1}, v_{2}, v_{3}, \ldots, v_{p}\right\} \bigcup\left\{w_{g}^{m}\right\}$, where $g=1,2,3, \ldots, p-1$ and $m=1,2,3, \ldots, s$. Let $W=\left\{w_{1}^{1}, w_{1}^{2}, w_{1}^{3}, \ldots, w_{1}^{s}\right\}$ be the resolving set of the $s$-Total $\left(P_{q}\right)$; then, $r\left(v_{g} \mid W\right)=(g, g-1, g-1, \ldots, g-1)_{(s \text {-tuple })}, \quad$ where $g=1,2,3, \ldots, p$, 


$$
r\left(w_{g}^{h} \mid W\right)= \begin{cases}(g-1, g, g, \ldots, g)_{(s-\text { tuple })} & g=1,2,3, \ldots, p-1 ; h=1, \\ (g, g-1, g, \ldots, g)_{(s \text {-tuple })} & g=1,2,3, \ldots, p-1 ; h=2, \\ (g, g, g-1, \ldots, g)_{(s-\text { tuple })} & g=1,2,3, \ldots, p-1 ; h=3, \\ \cdot & \\ (g, g, g, \ldots, g-1)_{(s-\text { tuple })}, & g=1,2,3, \ldots, p-1 ; h=s .\end{cases}
$$

When $g \neq m$ and $v_{g}$ and $v_{m}$ possess the same representation for $W$, a contradiction arises, i.e.,

$$
\begin{aligned}
r\left(v_{g} \mid W\right) & =r\left(v_{m} \mid W\right) \\
& \Rightarrow(g, g-1, g-1, \ldots, g-1)_{(s-\text { tuple })} \\
& =(m, m-1, m-1, \ldots, m-1)_{(s \text {-tuple })} \\
\Rightarrow g & =m(\text { contradiction }) .
\end{aligned}
$$

Similarly, when $l \neq m$,

$$
\begin{aligned}
r\left(w_{l}^{h} \mid W\right) & =r\left(w_{m}^{h} \mid W\right) \\
& \Rightarrow\left(l, l, l, \ldots, l-1_{h^{\text {th }}}\right. \text { position } \\
& l, \ldots, l)_{(s-\text { tuple })} \\
& =\left(m, m, m, \ldots, m-1_{h^{\text {th }}}\right. \text { position } \\
\Rightarrow l & m, \ldots, m)_{(s-\text { tuple })} \\
\Rightarrow l & =m \text { (contradiction) } .
\end{aligned}
$$

When $l \neq m$,

$$
\begin{aligned}
r\left(w_{g}^{l} \mid W\right) & =r\left(w_{g}^{m} \mid W\right) \\
& \Rightarrow\left(g, g, g, \ldots, g-1_{l^{\text {th }}} \text { position }, g \ldots, g\right)_{(s \text {-tuple })} \\
& =\left(g, g, g, \ldots, g-1_{m^{\text {th }} \text { position }}, g \ldots, g\right)_{(s \text {-tuple })} \\
\Rightarrow g-1 & =g(\text { contradiction }) .
\end{aligned}
$$

When $l \neq m$ and $u \neq v$,

$$
\begin{aligned}
r\left(w_{l}^{u} \mid W\right) & =r\left(w_{m}^{v} \mid W\right) \\
& \Rightarrow\left(l, l, l, \ldots, l-1{\left(u^{\text {th }} \text { position }\right)} l \ldots, l\right)_{(s-\text { tuple })} \\
& =\left(m, m, m, \ldots, m-1\left(_{\left.v^{\text {th }} \text { position }\right)}, m, \ldots, m\right)_{(s-\text { tuple })}\right. \\
\Rightarrow l-1 & =m \text { and } l=m-1(\text { contradiction }) .
\end{aligned}
$$

Now, we shall show that there is no resolving set with $s-1$ elements exists. So, on the contrary, let a set with $s-1$ elements be a resolving set for $s$-Total $P_{q}$. Then, particularly, for $s=3$, there exists a resolving set of $3-1=2$ elements. So, metric dimension of Three-Total $P_{q}$ is not 2, and it leads to contradiction.
So, there is no resolving set with $s-1$ elements, and hence, $s$-Total $\left(P_{q}\right)$ has metric dimension $s$.

1.3. Middle Tower Path Graph. Middle tower path graph is a special graph represented by Middle Tower $_{s}$, which is related to the path graph, and later, we extended its concept to its reflection.

Definition 3. An undirected, simple, and planar graph possessing vertex set $V=\left\{V_{g}^{t} \mid 1 \leq t \leq s, 1 \leq g \leq t\right\}$, where $t=1,2,3, \ldots, s$. Each vertex of Middle Tower $_{s}$ is adjacent in the following manner.

$$
\begin{aligned}
& \text { (1) } v_{g}^{t} \sim v_{g}^{t+1} \quad \text { when } \quad g=1,2,3, \ldots, t \quad \text { and } \\
& t=1, \stackrel{g}{2}, 3, \ldots, s-1 \\
& \text { (2) } v_{g}^{t} \sim v_{g+1}^{t+1} \quad \text { when } \quad g=1,2,3, \ldots, t \quad \text { and } \\
& t=1,2,3, \ldots, s-1 \\
& \text { (3) } v_{g}^{t} \sim v_{g+1}^{t} \quad \text { when } \quad g=1,2,3, \ldots, t \quad \text { and } \\
& t=1,2,3, \ldots, s-1
\end{aligned}
$$

Middle tower path graph is explained in Figure 5.

Theorem 7. Metric dimension of Middle Tower s is 1, for $s=2$.

Proof. As Middle Tower $_{2}$ is a path graph (by definition) and every path graph has metric dimension 1, so metric dimension of this graph is 1 .

Theorem 8. Metric dimension of Middle Tower is 2, for $s \geq 3$.

Proof. As Middle Tower $_{s}$ is not path graph; hence, it possesses metric dimension $\geq 2$. Vertex set of Middle Tower $_{s}$ is denoted by $V$ (Middle Tower $_{s}$ ) and is given by $V$ (Middle Tower $\left.P_{s}\right)=\cup_{p=1}^{s}\left\{h_{1}^{p}, h_{2}^{p}, h_{3}^{p}, \ldots, h_{q}^{p}\right\}, \quad$ where $q=1,2,3, \ldots, s-p+1$. We take $W=\left\{h_{1}^{1}, h_{1}^{s}\right\}$ resolving set of Middle Tower ${ }_{s}$; then, representation $r\left(h_{q}^{m} \mid W\right)=(q+m-$ $2, s-m), q=1,2,3, \ldots, s-m+1$ and $m=1,2,3, \ldots, s$.

When $g \neq r$ and $v_{g}$ and $v_{r}$ possess the same representation for $W$, a contradiction arises, i.e., 


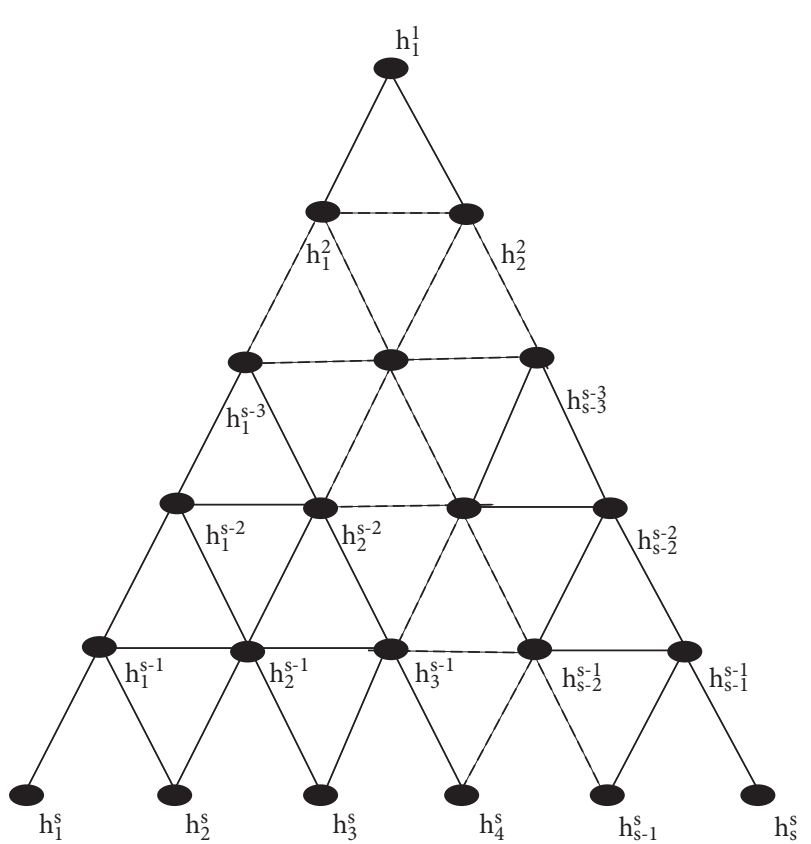

Figure 5: Middle tower path graph.

$$
\begin{aligned}
r\left(h_{q}^{m} \mid W\right) & =r\left(h_{r}^{m} \mid W\right) \\
\Rightarrow(q+m-2, s-m) & =(r+m-2, s-m) \\
\Rightarrow q & =r(\text { contradiction }) .
\end{aligned}
$$

Similarly, when $m \neq n$,

$$
\begin{aligned}
r\left(h_{q}^{m} \mid W\right) & =r\left(h_{q}^{n} \mid W\right) \\
\Rightarrow(q+m-2, s-m) & =(q+n-2, s-n) \\
\Rightarrow m & =n(\text { contradiction }) .
\end{aligned}
$$

When $m \neq n$ and $q \neq d$,

$$
\begin{aligned}
r\left(h_{q}^{m} \mid W\right) & =r\left(h_{d}^{n} \mid W\right) \\
\Rightarrow(q+m-2, s-m) & =(d+n-2, s-n) \\
\Rightarrow m & =n q=d(\text { contradiction }) .
\end{aligned}
$$

So, $W$ is the smallest resolving set for Middle Tower $P_{s}$ and its metric dimension is 2 .

1.4. Reflection Middle Tower Path Graph. Mirror image of Middle Tower $_{s}$ is named as Reflection Middle Tower $P_{s}$ and is denoted by $r l$ ( Tower $_{s}$ ).

Definition 4. Simple undirected graph possessing $V\left(r l\left(\right.\right.$ Tower $\left.\left._{s}\right)\right)=\left\{V_{(1) g}^{t} \mid 1 \leq t \leq s, 1 \leq g \leq t\right\} \bigcup\left\{V_{(2) g}^{t} \mid 1 \leq t\right.$ $\leq s, 1 \leq g \leq t\}$, where $t=1,2,3, \ldots, s$. Each vertex of $r l\left(\right.$ Tower $\left._{s}\right)$ is adjacent in the following manner.
(1) $v_{g}^{(i) t} \sim v_{g}^{(i) t+1}$ when $i=1,2, g=1,2,3, \ldots, t$, and $t=1,2,3, \ldots, s-1$

(2) $v_{g}^{(i) t} \sim v_{g+1}^{(i) t+1}$ when $i=1,2, g=1,2,3, \ldots, t$, and $t=1,2,3, \ldots, s-1$

(3) $v_{g}^{(i) t} \sim v_{g+1}^{(i) t}$ when $i=1,2, g=1,2,3, \ldots, t$, and $t=1,2,3, \ldots, s-1$

(4) $v_{g}^{(1) t} \sim v_{g+1}^{(2) t-1}$ when $g=1,2,3, \ldots, t-1$ and $t=s$

(5) $v_{g+1}^{(1) t} \sim v_{g}^{(2) t-1}$ when $g=1,2,3, \ldots, t-1$ and $t=s$

Reflection middle tower path graph is explained in Figure 6.

Theorem 9. Metric dimension of $r l\left(\right.$ Tower $\left._{s}\right)$ is 2 , for $s \geq 3$.

Proof. The vertex set of $r l\left(\right.$ Tower $\left._{s}\right)$ is $V\left(r l\left(\right.\right.$ Tower $\left.\left._{s}\right)\right)$ $=\left(\cup_{g=2}^{s-1}\left\{l_{1}^{(1) g}, l_{2}^{(1) g}, l_{3}^{(1) g}, \ldots, l_{j}^{(1) g}\right\} \quad \bigcup\left(\cup_{g=2}^{s-2}\left\{l_{1}^{(2) g}, l_{2}^{(2) g}\right.\right.\right.$, $\left.\left.\left.l_{3}^{(3) g}, \ldots, l_{j}^{(2) g}\right\}\right)\right)$, where $j=1,2,3, \ldots, s-g+1$.

Assume $W=\left\{l_{1}^{(1) g}, l_{2}^{(1) g}\right\}$ as the resolving set; then,

$$
r\left(l^{(1) 1_{j}} \mid W\right)= \begin{cases}(s-1, s-j+1), & j=1,2,3, \ldots, s-1, \\ (s-1,0), & j=s .\end{cases}
$$

$r\left(l_{j \mid W}^{(1) q}\right)_{=(s-q, s-j)}, \quad$ where $\quad j=1,2,3, \ldots, s-1 \quad$ and $q=2,3,4, \ldots, s$.

$r\left(l_{j \mid W}^{(2) q}\right)_{(s-q-2, s-j)}, \quad$ where $\quad j=1,2,3, \ldots, q \quad$ and $q=2,3,4, \ldots, s$.

When $m \neq n$ and $l_{m}$ and $l_{n}$ possess the same representation for $W$, a contradiction arises, i.e.,

$$
\begin{aligned}
r\left(l_{m}^{(1) 1} \mid W\right) & =r\left(l_{n}^{(1) 1} \mid W\right) \\
\Rightarrow(s-1, s-m+1) & =(s-1, s-n+1) \\
\Rightarrow m & =n(\text { contradiction }) .
\end{aligned}
$$

Similarly, when $m \neq n$,

$$
\begin{aligned}
r\left(l_{m}^{(1) h} \mid W\right) & =r\left(l_{n}^{(1) h} \mid W\right) \\
\Rightarrow(s-h, s-m) & =(s-h, s-n) \\
\Rightarrow m & =n \text { (contradiction). }
\end{aligned}
$$

When $m \neq n$

$$
\begin{aligned}
r\left(l_{p}^{(1) m} \mid W\right) & =r\left(l_{p}^{(1) n} \mid W\right) \\
\Rightarrow(s-m, s-1) & =(s-h, s-1) \\
\Rightarrow m & =n \text { (contradiction). }
\end{aligned}
$$

When $m \neq n$ and $u \neq v$,

$$
\begin{aligned}
r\left(l_{u}^{(1) m} \mid W\right) & =r\left(l_{v}^{(1) n} \mid W\right) \\
\Rightarrow(s-m, s-l) & =(s-n, s-v) \\
\Rightarrow m & =n \text { and } u=v(\text { contradiction }) .
\end{aligned}
$$




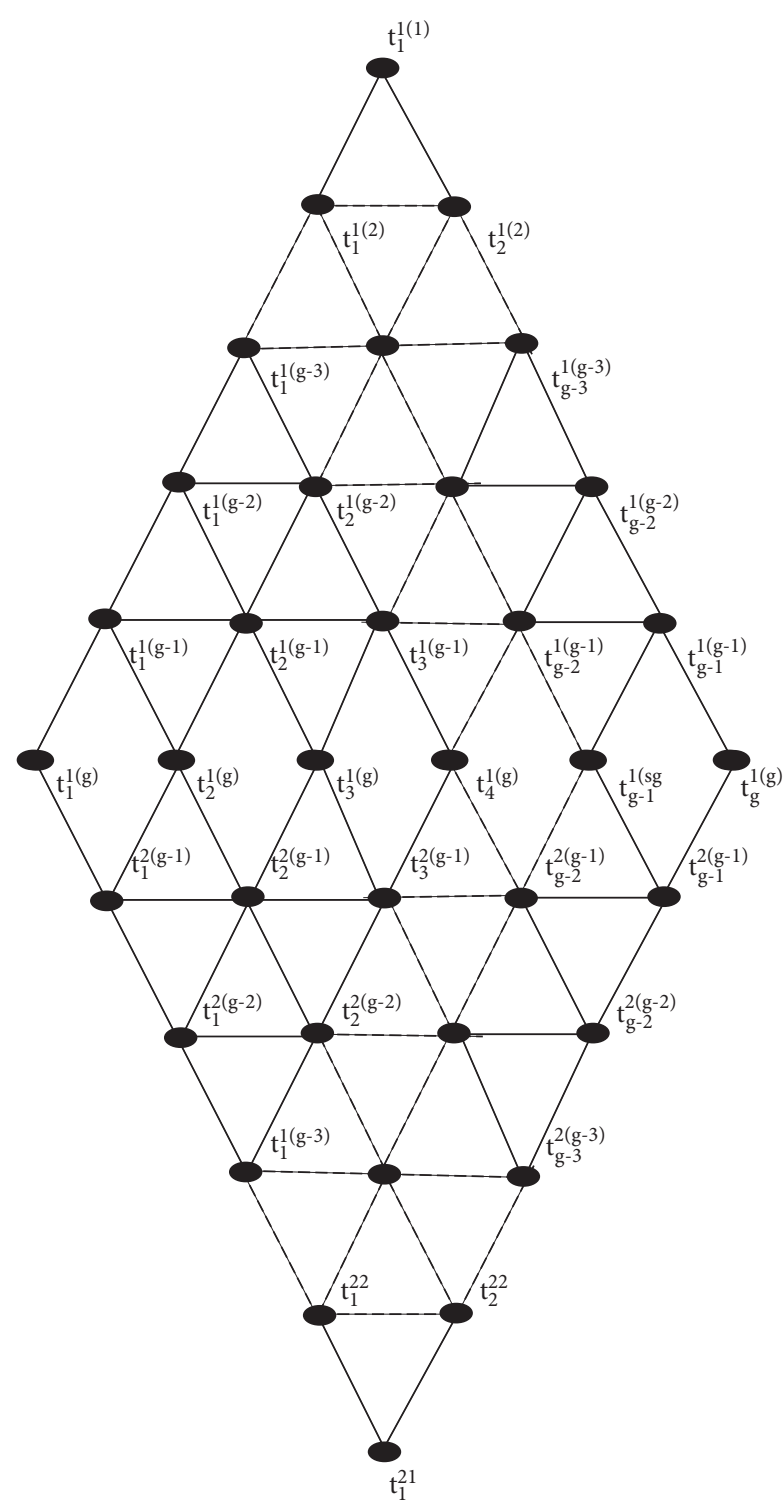

Figure 6: $r l\left(\right.$ Tower $\left._{s}\right)$ graph.

When $m \neq n$,

$$
\begin{aligned}
r\left(l_{m}^{(2) g} \mid W\right) & =r\left(l_{n}^{(2) g} \mid W\right) \\
\Rightarrow(s+g-2, s-m) & =(s+g-2, s-n) \\
\Rightarrow m & =n \text { (contradiction). }
\end{aligned}
$$

When $m \neq n$ and $u \neq v$,

$$
\begin{aligned}
r\left(l_{u}^{(2) m} \mid W\right) & =r\left(l_{v}^{(2) n} \mid W\right) \\
\Rightarrow(s+m-2, s-u) & =(s+n-2, s-v) \\
\Rightarrow m & =n \text { and } u=v(\text { contradiction }) .
\end{aligned}
$$

When $m \neq n$,

$$
\begin{aligned}
r\left(l_{c}^{(2) m} \mid W\right) & =r\left(l_{c}^{(2) n} \mid W\right) \\
\Rightarrow(s+m-2, s-c) & =(s+n-2, s-c) \\
\Rightarrow m & =n(\text { contradiction }) .
\end{aligned}
$$

Hence, $W$ is the smallest resolving set and metric dimension of $r l$ (Tower $\left._{s}\right)$ is 2 .

1.5. Symmetrical Planar Pyramid Graph. A graph is obtained by joining the neighbouring vertices of Middle Tower $P_{s}$ in its base and is represented by $\mathrm{SPP}_{s}$. If there are $s$ vertices in the base of $\mathrm{SPP}_{s}$, then its vertex set contains $s(s-1) / 2$ vertices.

Definition 5. We define $\mathrm{SPP}_{s}$ with $s$ vertices in base possesses vertex set $\cup_{t=1}^{s}\left\{V_{p}^{t}\right.$, where $t=1,2,3, \ldots, s$ and $p \leq t$.

Each vertex of tower ${ }_{s}$ is adjacent in the following manner.

(1) $v_{p}^{t} \sim v_{p}^{t+1} \quad$ when $\quad p=1,2,3, \ldots, s-1 \quad$ and $t=1,2,3, \ldots, s-1$ and $p \leq t$

(2) $v_{p}^{t} \sim v_{p+1}^{t+1} \quad$ when $\quad p=1,2,3, \ldots, s-1 \quad$ and $t=1,2,3, \ldots, s-1$

(3) $v_{p}^{t} \sim v_{p+1}^{t} \quad$ when $\quad p=1,2,3, \ldots, s \quad$ and $t=1,2,3, \ldots, s-1$

Symmetrical planar pyramid graph is explained in Figure 7.

Theorem 10. Metric dimension of $S P P_{s}$ is 2 .

Proof. The SPP has vertex set $V\left(\mathrm{SPP}_{s}=\cup_{t=1}^{s}\right.$, where $p=$ $1,2,3, \ldots s$ and $t=1,2,3, \ldots, s$. Also, $p \leq t$.

We take $W=\left\{v_{1}^{1}, v_{s}^{s}\right\}$ resolving set of Middle $\operatorname{SPP}_{s}$ and then representation

$r\left(v_{p}^{t} \mid W\right)=(p+j-1, s-t+j) p=1,2,3, \ldots, s \quad$ and $t=1,2,3, \ldots, s$. Also, $p \leq t$.

When $l \neq g$ and $v_{l}$ and $v_{g}$ possess the same representation for $W$, a contradiction arises, i.e.,

$$
\begin{aligned}
r\left(v_{l}^{t} \mid W\right) & =r\left(v_{g}^{t} \mid W\right) \\
\Rightarrow(l+j-1, s-t+j) & =(g+j-1, s-t+j) \\
\Rightarrow l & =g \text { (contradiction) } .
\end{aligned}
$$

Similarly, when $l \neq g$,

$$
\begin{aligned}
r\left(v_{p}^{l} \mid W\right) & =r\left(v_{p}^{g} \mid W\right) \\
\Rightarrow(p+j-1, s-l+j) & =(p+j-1, s-g+j) \\
\Rightarrow l & =g(\text { contradiction }) .
\end{aligned}
$$

When $l \neq g$ and $u \neq v$, 


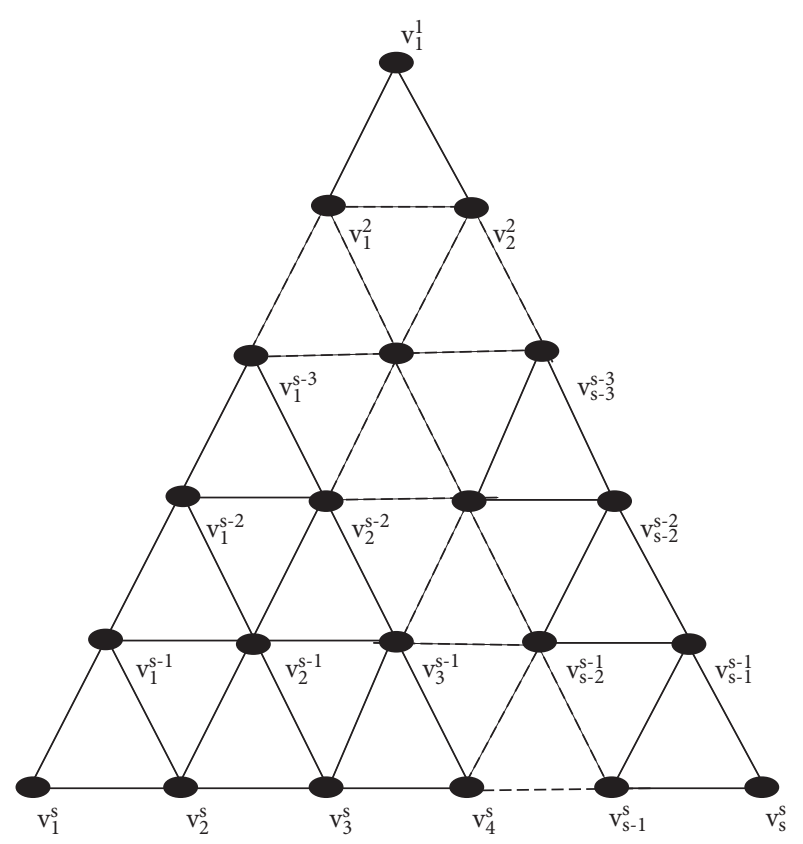

Figure 7: Symmetrical planar pyramid graph.

$$
\begin{aligned}
r\left(v_{u}^{l} \mid W\right) & =r\left(v_{v}^{g} \mid W\right) \\
\Rightarrow(l+j-1, s-u+j) & =(g+j-1, s-v+j) \\
\Rightarrow l & =g u=v \text { (contradiction). }
\end{aligned}
$$

So, $W$ is the smallest resolving set for $\mathrm{SPP}_{s}$ and its metric dimension is 2 .

1.6. Reflection Symmetrical Planar Pyramid Graph. Mirror image of $\mathrm{SPP}_{s}$ is named as reflection symmetrical planar pyramid graph and is denoted by $r l\left(\mathrm{SPP}_{s}\right)$.

Definition 6. Simple undirected graph possessing vertex set $V\left(r l\left(\mathrm{SPP}_{s}=\left(\cup_{t=1}^{s} V_{p}^{(1) t}\right) \bigcup\left(\cup_{t=2}^{s-1} V_{p}^{(2) t}\right)\right.\right.$, where $p=1,2,3, \ldots, s$ and $t=1,2,3, \ldots, s$; also, $s \leq t$. Each vertex of $r l\left(\mathrm{SPP}_{s}\right)$ is adjacent in the following manner.
(1) $v_{p}^{(i) t} \sim v_{p}^{(i) t+1} \quad$ when $\quad p=1,2,3, \ldots, s$, $t=1,2,3, \ldots, s-1, p \leq t$, and $i=1,2$
(2) $v_{p}^{(i) t} \sim v_{p+1}^{(i) t+1} \quad$ when $\quad p=1,2,3, \ldots, s$, $t=1,2,3, \ldots, s-1, p \leq t$, and $i=1,2$
(3) $v_{p}^{(i) t} \sim v_{p}^{(i) t} \quad$ when $\quad p=1,2,3, \ldots, s$, $t=1,2,3, \ldots, s-1, p \leq t$, and $i=1,2$
(4) $v_{p}^{(1) t} \sim v_{p}^{(2) t-1}$ when $t=s$ and $g=2,3,4, \ldots, s$
(5) $v_{p+1}^{(1) t} \sim v_{p}^{(2) t-1}$ when $t=s$ and $p=1,2,3, \ldots, s-1$

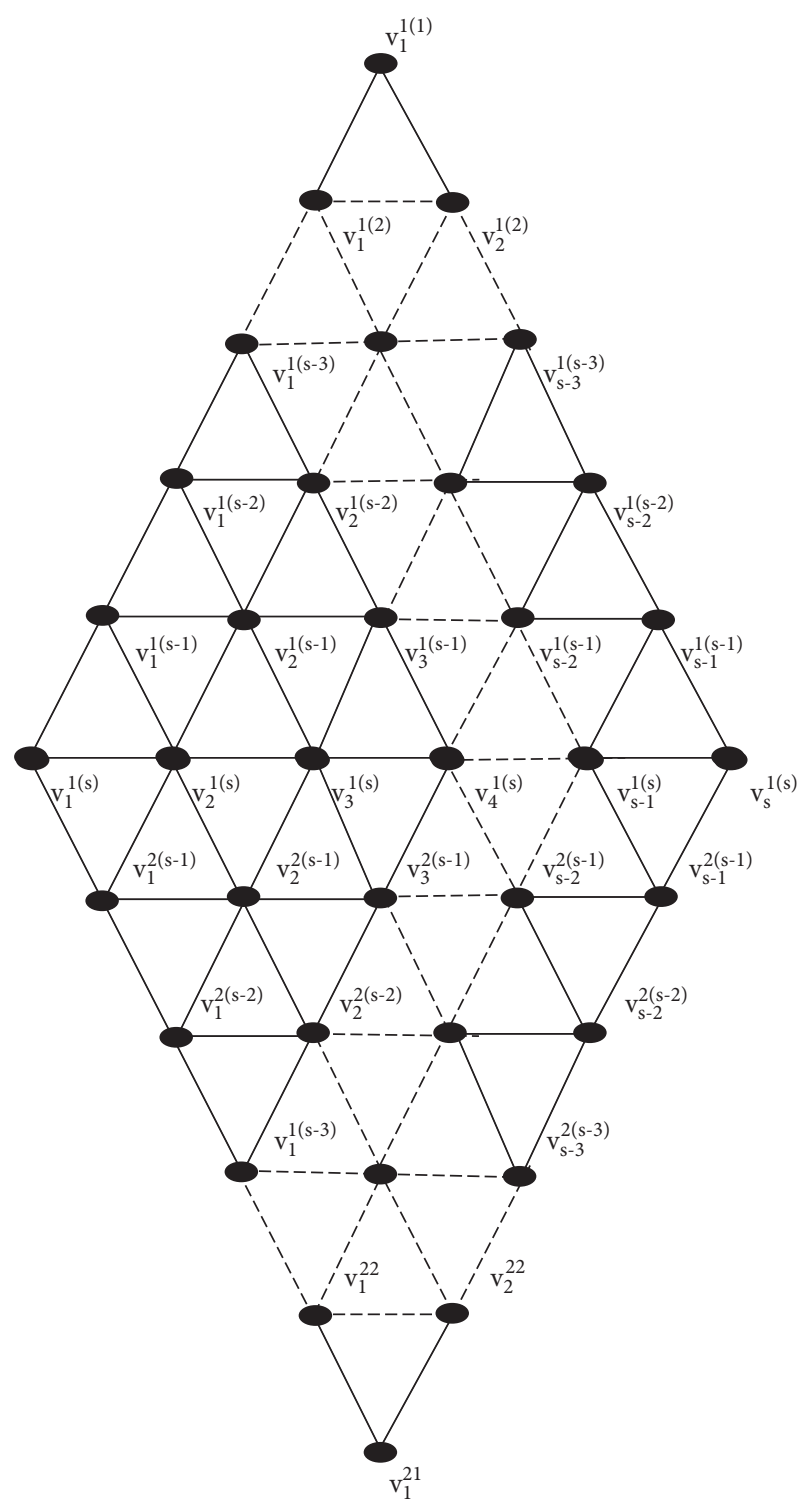

FIgURE 8: Reflection symmetrical planar pyramid graph.

Reflection symmetrical planar pyramid graph is explained in Figure 8.

Theorem 11. Metric dimension of $r l\left(S P P_{s}\right)$.

Proof. The vertex set of $r l\left(\mathrm{SPP}_{s}\right)$ is $V\left(r l\left(\operatorname{SPP}_{s}\right)\right)=\left(\cup_{t=2}^{s}\right) \bigcup\left(\cup_{t=1}^{s-1} V_{p}^{(2) t}\right), \quad$ where $p=1,2,3, \ldots, s$ and $t=1,2,3, \ldots, s$; also, $s \leq t$.

Assume $W=\left\{v_{1}^{(1)}, v_{s}^{s}\right\}$ as the resolving set; then,

$$
\begin{aligned}
& r\left(v^{(1) t_{p}} \mid W\right)=\{(p-j-1, s-(t+1 J)), \quad p=1,2,3, \ldots, s t=1,2,3, \ldots, s p \leq t \text { and } j=t-p, \\
& r\left(v^{(2) t_{p}} \mid W\right)=\{(s-t+p-1,2 s-t-1), \quad j=1,2,3, \ldots, s-1 .
\end{aligned}
$$


When $l \neq g$ and $v_{l}$ and $v_{g}$ possess the same representation for $W$, a contradiction arises, i.e.,

$$
\begin{aligned}
r\left(v_{l}^{(1) t} \mid W\right) & =r\left(v_{g}^{(1) t} \mid W\right) \\
\Rightarrow(l+j-1, s-t+j) & =(g+j-1, s-g+j) \\
\Rightarrow l & =g(\text { contradiction }) .
\end{aligned}
$$

Similarly, when $l \neq g$,

$$
\begin{aligned}
r\left(v_{p}^{(1) l} \mid W\right) & =r\left(v_{p}^{(1) g} \mid W\right) \\
\Rightarrow(p+j-1, s-l+j) & =(p-j-1, s-g+j) \\
\Rightarrow l & =g \text { (contradiction). }
\end{aligned}
$$

When $l \neq g$,

$$
\begin{aligned}
r\left(v_{l}^{(1) u} \mid W\right) & =r\left(v_{g}^{(1) p} \mid W\right) \\
\Rightarrow(l+j-1, s-u+j) & =(g+j-1, s-p+j) \\
\Rightarrow l & =g u=p \text { (contradiction). }
\end{aligned}
$$

When $l \neq g$,

$$
\begin{aligned}
r\left(v_{l}^{(2) t} \mid W\right) & =r\left(v_{g}^{(2) t} \mid W\right) \\
\Rightarrow(s-t+l-1,2 s-t-l) & =(s-t+g, 2 s-t-1) \\
\Rightarrow l & =g(\text { contradiction }) .
\end{aligned}
$$

When $l \neq g$,

$$
\begin{aligned}
r\left(v_{p}^{(2) t} \mid W\right) & =r\left(v_{p}^{(2) g} \mid W\right) \\
\Rightarrow(s-l+p-1,2 s-t-1) & =(s-g+p-1,2 s-t-1) \\
\Rightarrow l & =g(\text { contradiction }) .
\end{aligned}
$$

When $l \neq g$ and $u \neq p$,

$$
\begin{aligned}
r\left(v_{l}^{(2) u} \mid W\right) & =r\left(v_{g}^{(2) p} \mid W\right) \\
\Rightarrow(s-u+l-1,2 s-u-1) & =(s-v+g-1,2 s-p-1) \\
\Rightarrow l & =g \text { and } u=p \text { (contradiction). }
\end{aligned}
$$

Hence, $W$ is the smallest resolving set and metric dimension of $r l\left(\mathrm{SPP}_{s}\right)$ is 2 .

\section{Conclusions}

In this dissertation, some new graphs as $s-\operatorname{Mid}(G)$, $s-\operatorname{Total}(G)$, Middle Tower $_{s}, \mathrm{SPP}_{s}, r l\left(\right.$ Tower $\left._{s}\right)$, and $r l\left(\mathrm{SPP}_{s}\right.$ are defined and metric dimension of these graphs is also computed. All these graphs possess constant and bounded metric dimension. This research work may lead to the following open problems.

Open problem 1: compute metric dimension of symmetrical pyramid in space.

Open problem 2: to find metric dimension of $s-\operatorname{Mid}\left(C_{n}\right)$

Open problem 3: to find metric dimension of $s-T\left(C_{n}\right)$

\section{Data Availability}

No data were used to support this study.

\section{Disclosure}

The paper has not been published elsewhere, and it will not be submitted anywhere else for publication.

\section{Conflicts of Interest}

The authors declare that they have no conflicts of interest.

\section{Authors' Contributions}

Each author has equal contribution.

\section{Acknowledgments}

The authors extend their appreciation to the Deanship of Scientific Research at Majmaah University for funding this work, under project no. RGP-2019-29.

\section{References}

[1] P. J. Slater, "Leaves of trees," Congressus Numerantium, vol. 14, pp. 549-559, 1975.

[2] F. Harary and R. A. Melter, "On the metric dimension of a graph,” Ars Combinatoria, vol. 2, pp. 191-195, 1976.

[3] R. Naeem and M. Imran, "On resolvability and exchange property in antiweb-wheels," Utilitas Mathematica, vol. 104, pp. 187-200, 2017.

[4] S. Khuller, B. Raghavachari, and A. Rosenfeld, "Localization in graphs," vol. 94-92, University of Maryland, College Park, MD, USA, 1994, Tech. Rep. UMIACS-TR.

[5] J. Kratica, V. Kovačević-Vujčić, M. Stojanović, and M. Stojanovic, "Minimal doubly resolving sets and the strong metric dimension of some convex polytopes," Applied Mathematics and Computation, vol. 218, no. 19, pp. 97909801, 2012.

[6] M. Perc, J. Gómez-Gardeñes, A. Szolnoki, L. M. Floría, and Y. Moreno, "Evolutionary dynamics of group interactions on structured populations: a review," Journal of The Royal Society Interface, vol. 10, no. 80, Article ID 20120997, 2013.

[7] M. Perc and A. Szolnoki, "Coevolutionary games-A mini review," Biosystems, vol. 99, no. 2, pp. 109-125, 2010.

[8] A. Szolnoki and M. Perc, "Correlation of positive and negative reciprocity fails to confer an evolutionary advantage: phase transitions to elementary strategies," Physical Review X, vol. 3, no. 4, Article ID 041021, 2013.

[9] I. G. Yero, D. Kuziak, and J. A. Rodríguez-Velázquez, "On the metric dimension of corona product graphs," Computers \& Mathematics with Applications, vol. 61, no. 9, pp. 2793-2798, 2011.

[10] M. Ali, M. T. Rahim, and G. Ali, "On path related graphs with constant metric dimension," Utilitas Mathematica, vol. 88, no. 1, pp. 203-209, 2012.

[11] G. Chartrand, L. Eroh, M. A. Johnson, and O. R. Oellermann, "Resolvability in graphs and the metric dimension of a graph," Discrete Applied Mathematics, vol. 105, no. 1-3, pp. 99-113, 2000. 
[12] M. Imran, A. Q. Baig, S. A. U. H. Bokhary, and I. Javaid, "On the metric dimension of circulant graphs," Applied Mathematics Letters, vol. 25, no. 3, pp. 320-325, 2012.

[13] M. Imran, M. K. Siddiqui, and R. Naeem, "On the metric dimension of generalized petersen multigraphs," IEEE Access, vol. 6, pp. 74328-74338, 2018.

[14] R. A. Melter and I. Tomescu, "Metric bases in digital geometry," Computer Vision, Graphics, and Image Processing, vol. 25, no. 1, pp. 113-121, 1984.

[15] S. W. Saputro, R. Simanjuntak, S. Uttunggadewa et al., "The metric dimension of the lexicographic product of graphs," Discrete Mathematics, vol. 313, no. 9, pp. 1045-1051, 2013.

[16] P. J. Slater, "Dominating and reference sets in a graph," Journal of Mathematical and Physical Sciences, vol. 22, no. 4, pp. 445-455, 1988.

[17] M. M. Alholi, O. A. Abughneim, and H. A. Ezeh, "Metric dimension of some path related graphs," Global Journal of Pure and Applied Mathematics, vol. 3, no. 2, pp. 149-157, 2017.

[18] A. T. Shahida and M. S. Sunitha, "On the metric dimension of joins of two graphs," Global Journal of Pure and Applied Mathematics, vol. 5, no. 9, pp. 33-38, 2014.

[19] H. Peng, X. Wang, J. Wang, and J. Chen, "On the metric dimension of some Kneser graphs," Journal of Computational and Theoretical Nanoscience, vol. 13, no. 5, pp. 3013-3018, 2016.

[20] H. Pan, M. Ali, G. Ali, M. T. Rahim, and X. Yang, "On the families of graphs with unbounded metric dimension," IEEE Access, vol. 7, pp. 165060-165064, 2019.

[21] S. Nawaz, M. Ali, M. A. Khan, and S. Khan, "Computing metric dimension of power of total graph," IEEE Access, vol. 9, pp. 74550-74561, 2021. 